\title{
Micro Vapor Cells Sealed by Two-step Bonding for Miniature Atomic Clocks
}

Hitoshi Nishino ( $\square$ nishino-hitoshi@tmex.co.jp )

Tamagawa Holdings Co.,Ltd

\section{Yasubumi Furuya}

Tohoku University

\section{Takahito Ono}

Tohoku University

\section{Research Article}

Keywords: Micro Vapor Cells, Two-step Bonding, Miniature Atomic Clocks, proposal, fabrication

Posted Date: July 8th, 2021

DOl: https://doi.org/10.21203/rs.3.rs-686573/v1

License: (c) (i) This work is licensed under a Creative Commons Attribution 4.0 International License. Read Full License 


\title{
Micro vapor cells sealed by two-step bonding for miniature atomic clocks
}

Hitoshi Nishino, a), Yasubumi Furuya ${ }^{2}$, and Takahito Ono ${ }^{2,3}$

${ }^{1}$ Tamagawa Holdings Co.,Ltd., Tokyo, 105-0013, Japan

${ }^{2}$ Micro System Integration Center, Tohoku University, Sendai, Miyagi 980-0845, Japan

${ }^{3}$ Department of Mechanical Systems Engineering, Graduate school of Engineering, Tohoku University, Sendai, Miyagi 980-8579, Japan

${ }^{a)}$ Author to whom correspondence should be addressed: nishino-hitoshi@tmex.co.jp

\begin{abstract}
This paper reports the proposal, fabrication, and evaluation of $\mathrm{Rb}$ vapor cells sealed by two-step bonding for miniature atomic clocks. The proposed fabrication method is separating the light path filled with Alkali atoms and Alkali dispenser's room at wafer level process. The first fabricated sample is unexpectedly sealed with some air, and the vapor cell is measured the absorption spectra and CPT resonance. The flow solving the problems is indicated, and the vapor cells are fabricated again with the improved process. The FWHM of CPT resonance fits almost the theoretical value in vacuum-sealed samples, which are filled with $\mathrm{Rb}$ atoms carried from one dispenser. These results show that fabricated vapor cells are filled with $\mathrm{Rb}$ without degassing or leaks, and the reliability of the fabrication process and the production efficiency can be expected. Rb vapor cell sealed in the controlled pressure with buffer gases $\mathrm{N}_{2} / \mathrm{Ar}$ is also fabricated and evaluated. The spectrum line is broadened, and CPT resonance peak is narrowed $4.0 \mathrm{kHz}$ at $90{ }^{\circ} \mathrm{C}$. These results show the proposal fabrication method of the alkali vapor cells is useful for miniature atomic clocks.
\end{abstract}




\section{Introduction}

The demand of larger amount of data and higher speed communication are always existed. To meet the demand, carrier waves are employed to a wider range or higher frequency, and precise time synchronization system have been developed. In the time synchronization for communication, the base station is needed to have exact time and have atomic clocks. Recently miniature atomic clocks are expected to put on mobile devices like smartphone in order to have accurate time synchronization between the terminal and base station, or terminal and another terminal ${ }^{1-10}$.

Miniature atomic clocks have vapor cells, which are including alkali atoms $\mathrm{Rb}$ or $\mathrm{Cs}^{1,2}$. Applying the laser light to the vapor cell, the reference signal based on the gap of the energy level of the hyperfine structure of Alkali atoms can be obtained from the transmitted light. The reference signal is used to correct the error of the oscillator. This oscillator can keep precise time, and such feedback system is atomic clocks ${ }^{11-14}$. In addition, recently, the subpicotesla magnetometry or NMR gyroscope using alkali vapor cells has developed ${ }^{15-19}$.

The vapor cells are formed by the microfabrication methods ${ }^{20-22}$. Sealing is used anodic bonding at the wafer-level ${ }^{23}$. There have been developed the novel fabrication methods to seal with Alkali atoms, but still it is challenging to seal Alkali atoms at wafer-level packaging ${ }^{24}$. With the mixture of $\mathrm{AlkaliCl}$ and $\mathrm{BaN}_{6}{ }^{25}, \mathrm{Rb}$ can be evaporated each cell from the glass ample, and the anodic bonding with a glass wafer is performed in the chamber. This method is needed complicated machines and the cost of the vapor cell is increased. With the alkali atom dispensers, the fabrication process can be straightforward, but the space of the dispenser is needed ${ }^{4,26,27}$. This method refuses miniaturization, and $\mathrm{Al} / \mathrm{Zr}$ can absorb $\mathrm{N}_{2}$ gas, which is widely used as buffer gas $^{28}$. 


\section{The principle and calculation method for CPT resonance}

Figure 1 (a) shows the coherent population trapping (CPT) system, which is normally chosen for miniature atomic clock operation ${ }^{11,29-31}$. CPT is induced by irradiating two laser fields, which are obtained as sidebands from the modulated laser. When the energy difference of the two laser fields is equal to the energy gap of the two ground states, quantum interference impedes photo-absorption, resulting in a narrow transmission peak, which is known as the CPT resonance. By locking the peak, the oscillator's signal can be corrected and keep stable. The short-term frequency stability of the atomic clocks is often characterized by Allan deviation $\sigma_{y}$, which is expressed by

$$
\sigma_{y} \propto \frac{1}{Q \cdot(S / N)} \tau^{-1 / 2}
$$

where $Q$ is the quality factor of the resonance peak, $\tau$ is averaging time, $S / N$ is the signal-to-noise ratio in detecting the CPT resonance ${ }^{32}$. Q is defined as the ratio of the resonant frequency $f_{r f}$ and FWHM (Full Width Half Maximum) of the CPT resonant peak $\Delta v$, which is express as

$$
Q=\frac{f_{r f}}{\Delta v}
$$

$\Delta v$ is given by

$$
\Delta v=\Gamma_{L}=\frac{1}{\pi}\left(\gamma_{2}+\omega_{R}^{2} / \Gamma^{*}\right)
$$

, where $\gamma_{2}$ is the relaxation rate in the absence of laser radiation, $\omega_{R}$ is Rabi angular frequency, and $\Gamma^{*}$ is the decay rate of the excited state. It is known that $\omega_{R}^{2} / \Gamma^{*}$ is proportional to the laser intensity ${ }^{29} \cdot \gamma_{2}$ is described as the sum of the relaxation by wall collision $\gamma_{2 w}$, the relaxation by $\mathrm{Rb}$ atoms with buffer-gas molecule $\gamma_{2 b g}$, and spinexchange interaction between $\mathrm{Rb}$ atoms $\gamma_{2 s e}$, as follows 


$$
\gamma_{2}=\gamma_{2 w}+\gamma_{2 b g}+\gamma_{2 s e}
$$

$\gamma_{2 w}$ is given as

$$
\gamma_{2 w}=\left[\left(\frac{2.405}{a}\right)^{2}+\left(\frac{\pi}{L}\right)^{2}\right] D_{0} \frac{P}{P_{0}}\left(\frac{T}{T_{0}}\right)^{3 / 2}
$$

, where $a$ is the radius, $L$ is the length of the cylindrical column, $D_{0}$ is diffusion coefficient, $P$ is buffer gas pressure at temperature $T, P_{0}$ is reference pressure at a temperature $T_{0}$. The cylindrical column image shows in Fig. 1 (b). $\gamma_{2 b g}$ is given as

$$
\gamma_{2 b g}=N_{0} \sigma_{b u f f e r} \bar{v}_{r e l}\left(\frac{p}{p_{0}}\right)
$$

, where $N_{0}$ is Loschmidt's constant, $\sigma_{\text {buffer }}$ is disorientation cross-section which characterizes rubidium-buffer gas collisions, $\bar{v}_{r e l}$ is the mean relative velocity of rubidium and buffer gas atoms. $\gamma_{2 e x}$ is given as

$$
\gamma_{e x}=\frac{6 I+1}{8 I+4} N_{0} \sigma_{e x} \bar{v}_{r}
$$

, where $I$ is nuclear spin quantum number, $\sigma_{e x}$ is disorientation cross-section which characterize rubidium atom collisions.

The buffer gases are used to reduce the relaxation due to the wall collisions. Without the buffer gases, the main relaxation depends on the wall collision. The relation is express as

$$
\Delta v / 2 \approx \frac{v_{R b}}{L_{\text {mean }}}
$$

where $v_{R b}$ is the $1 / e$ velocity width of a Maxwell Boltzmann distribution and $L_{m e a n}$ is the mean free path of $\mathrm{Rb}$ atom, and $L_{\text {mean }}$ is almost same as the minimum length in the vapor cell ${ }^{33}$. From this equation, FWHM of CPT resonance in the vacuum-sealed vapor cell can be calculated. The vapor cells in this paper are designed $L=2.0 \mathrm{~mm}$ and $a=1.0$ $\mathrm{mm}$. For miniature atomic clocks, FWHM of the CPT resonance can be calculated 1.9 $\mathrm{kHz}$ with N2/Ar buffer gases $3.6 \mathrm{kPa}$ at $90{ }^{\circ} \mathrm{C}$. 


\section{Proposal vapor cells and fabrication}

Figure 2 show the conventional-typed vapor cells using Alkali-atom dispenser and the proposal vapor cell, which is filled with $\mathrm{Rb}$ from Alkali-atom dispenser. What you can see from the viewpoint of the structural feature is that an Alkali-atom dispenser remains in Fig. 2 (a), on the other hand, an Alkali-atom dispenser is detached in Fig. 2 (b). There are many fabrication methods to use glass wafer ${ }^{34,35}$, and in this process, by etching the glass $\mathrm{Rb}$ atoms can be moved through the gap and the small gap can be sealed by anodic bonding after first sealing.

Figure 3 (a) shows the process chart of the proposal vapor cell. (Process 1) 2 mm-thick Si wafer is processed hole by mechanical drills. (Process 2) Anodic bonding is performed with $1 \mathrm{~mm}$-thick Tempax glass. The temperature was $450{ }^{\circ} \mathrm{C}$ and the applied voltage was $-600 \mathrm{~V}$ under the atmosphere for $5 \mathrm{~min}$. (Process 3) After filling the Rb dispenser (SAES Getters RB/AMAX/PILL/1-0.6), anodic bonding is performed with $1 \mathrm{~mm}$-thick Tempax glass, where the center part is etched $2.0 \mu \mathrm{m}$ in depth by DHF (Diluted Hydrogen Fluorine). The bonding temperature was $400{ }^{\circ} \mathrm{C}$, and the applied voltage was $-450 \mathrm{~V}$ for 7 min in a vacuum or the controlled pressure with buffer gases. (Process 4) Activation of the $\mathrm{Rb}$ dispenser is performed by $\mathrm{YAG}$ laser. $\mathrm{Rb}$ atoms can move each cell during increasing the temperature for anodic bonding. The center part is anodically bonded. The temperature was $400{ }^{\circ} \mathrm{C}$, and the applied voltage was $-900 \mathrm{~V}$ under the atmosphere for 5 min. (Process 5) Dicing is performed. One of the vapor cells is sketched up.

Figure 3 (b) shows the $20 \mathrm{~mm}$-square wafer after Process 3, and only the surrounding was founded to be anodically bonded, because the changed color of the bonded area and center of the wafer was curved because the difference of the pressure between in the cell 
and outside and the difference of the coefficient of thermal expansion between the Si and Tempax glasses ${ }^{36,37}$. Figure 3 (c) shows the wafer after Process 4, and the center part was anodically-bonded and cells were sealed with $\mathrm{Rb}$ metal successfully. Deposited Rb was observed at the glass surface of some cells. Figure 3 (d) shows the vapor cell after Process 5. $3.7 \times 3.7 \times 4.0 \mathrm{~mm}$ vapor cell was picked up.

\section{Evaluation and feedback}

The evaluation setup for the Rb absorption spectrum is shown in Fig. 4 (a), and for CPT resonance is shown in Fig. 4 (b). In this measurement, VCSEL of $795 \mathrm{~nm}$ wavelength is used. A photodiode was used for the detection of the transmitted laser beam. In order to shield external magnetic fields, the vapor cell was covered with permalloy plates.

Figure 4 (c) shows the absorption spectrum of the fabricated vacuum sealed $\mathrm{Rb}$ vapor cell at $90{ }^{\circ} \mathrm{C}$. There are $85 \mathrm{Rb}$ and $87 \mathrm{Rb}$ with natural abundance in the vapor cells, and four $87 \mathrm{Rb}$ absorption line were observed. Comparing the theoretical $\mathrm{Rb}$ spectrum in vacuum-sealed $\mathrm{Rb}$ vapor cell, absorption lines were broadened ${ }^{38}$. Figure 4 (d) shows the CPT resonant peak, which was shifted $4.7 \mathrm{kHz}$ from the transition frequency for $87 \mathrm{Rb}$, 6.83 GHz shown in Fig. 1 (a). This shift indicates there are some gases in the vapor cell, and FWHM of the peak was $4.3 \mathrm{kHz}$. These results meant unexpected gases remains, but the remained gases were worked as the buffer gases very well. It seemed the gases were from the air through the small holes between the Tempax glass and Si surface after Process 3 in Fig. 3 (a). If the leak occurs after Process 5, Rb in the vapor cell can react with much oxygen in air and $\mathrm{Rb}$ atoms would be oxidized and the absorption spectra wouldn't be obtained. 
Figure 5 (a) shows the Si wafer after Process 1 in Fig. 3 (a), and the hole processed by mechanical drills is observed by SEM (Scanning Electron Microscope) in Fig. 5 (b). Figure 5 (c) and (d) were also observed by SEM, and the scratches were found on the Si surface. Those scratches were possibly formed in the mechanical drill process. Figure 5 (e) and (f) show that the air sealing process and expected phenomenon. Air includes approximately $80 \% \mathrm{~N}_{2}$ and $20 \% \mathrm{O}_{2}$, and $\mathrm{O}_{2}$ can be absorbed by the Rb dispenser. $\mathrm{N}_{2}$ cannot be easily absorbed by the $\mathrm{Rb}$ dispenser and can behave as a buffer gas.

In order to smooth the scratches, a polishing process was added after Process 1 in Fig. 3 (a). Then we fabricated and evaluated three vapor cells; two are sealed in vacuum in Process 3, and the other is sealed in the controlled pressure with buffer gases $\mathrm{N}_{2} / \mathrm{Ar}$, which mix ratio is $\mathrm{N}_{2}: A r=5: 8$. Figure 6 (a) shows the vacuum sealed $20 \mathrm{~mm}$-square wafer after Process 3 in Fig. 3 (a), and only the surrounding was founded to be anodically bonded. Figure 6 (b) shows the wafer after Process 4, and the center part was anodicallybonded and cells were sealed. Deposited Rb was also observed at the glass surface of some cells. Figure 6 (c) shows the vapor cell after Process 5. $3.7 \times 3.7 \times 4.0 \mathrm{~mm}$ vapor cell was picked up.

$\mathrm{Rb}$ absorption spectra of the two vapor cells, which were vacuum sealed were shown in Fig. 6 (d). There are $85 \mathrm{Rb}$ and $87 \mathrm{Rb}$ with natural abundance in the vapor cells, and four $87 \mathrm{Rb}$ absorption lines were observed. The vapor cells were heated up to $90{ }^{\circ} \mathrm{C}$, and two samples showed almost same spectrum lines. Comparing the theoretical Rb spectrum in vacuum-sealed $\mathrm{Rb}$ vapor cell, absorption lines were not broadened ${ }^{38}$. The CPT resonance spectra of two vapor cells were shown in Fig. 6 (e). The vapor cells were heated up to $90{ }^{\circ} \mathrm{C}$ and a weak magnetic field was applied for Zeeman shift. The FWHM/2 of the peaks were $95 \mathrm{kHz}$ and $110 \mathrm{kHz}$, respectively. The theoretical value estimated from equation 
(8) is $131 \mathrm{kHz}$, and comparing the values, a little gas remains and behaves as buffer gases $^{39}$. The pressure was predicted from equation (6), and the value were around several dozen $\mathrm{Pa}$ in both vapor cells. These are the minimal numbers, and the result means this proposal fabrication method is very reliable, because almost no degassing and leak were found.

Figure 7 (a) shows the $\mathrm{Rb}$ absorption spectrum of the vapor cell sealed with controlled pressure of the buffer gases $\mathrm{N}_{2} / \mathrm{Ar}$. The $\mathrm{Rb}$ spectrum lines was found broadened by buffer gases. Figure 7 (b) shows the measured CPT resonance, and the peak was shifted by 3.6 $\mathrm{kHz}$ from the clock frequency, and $\mathrm{N}_{2} / \mathrm{Ar}$ buffer gasses pressure was estimated to be 2.76 $\mathrm{kPa}^{11}$. The pressure was expected to seal $3.6 \mathrm{kPa}$ at $90{ }^{\circ} \mathrm{C}$ in the fabrication process, and this difference can be considered because of the unevenness of the temperature and pressure in the chamber during anodic bonding in Process 3 in Fig. 3 (a). The FWHM of the CPT resonance peak was $4.0 \mathrm{kHz}$ at $90{ }^{\circ} \mathrm{C}$, and $\mathrm{FWHM}$ from a theoretical calculation using the cylindrical cavity model from the equation (4), (5), (6), and (7) was $2.1 \mathrm{kHz}$. The difference might be caused by the power broadening. Although there were some differences from theoretical values, it can be said that the vapor cell fabricated successfully for miniature atomic clocks.

\section{Summary}

In summary, the proposal, fabrication, and evaluation of $\mathrm{Rb}$ vapor cells sealed by twostep bonding for miniature atomic clocks were introduced. First fabricated vapor cell had some problems, but the result was better for miniature atomic clocks. There was almost no reproducibility, but it can be said that this is the vapor cell using some atmosphere as buffer gases. Then the problem was expected that some scratches during mechanical drills, 
which resulted in leak, and a polishing process was added in the proposal fabrication process. By the improved fabrication process, two vacuum-sealed vapor cells had almost theoretical absorption spectra and CPT resonant peaks, and the degassing and leak were found to be minimal. The proposal fabrication process is separating the light path room with $\mathrm{Rb}$ and dispenser's room, and additionally the fabricated two vapor cells filled with $\mathrm{Rb}$ from one $\mathrm{Rb}$ dispenser indicated the lower cost fabrication process. The vapor cell sealed in the controlled pressure with buffer gases $\mathrm{N}_{2} / \mathrm{Ar}$ was measured the absorption spectra and CPT resonance. The FWHM of the CPT resonance was narrowed $4.0 \mathrm{kHz}$ at $90{ }^{\circ} \mathrm{C}$. These results show the proposal fabrication method of the alkali vapor cells is widely useful for miniature atomic clocks. 


\section{Acknowledgments}

Part of this research was performed at Tohoku University Micro System Integration Center and Micro / Nano-Machining Research and Education Center.

The measurement of the absorption ratio and CPT resonance were performed in NICT (National Institute of Information and Communications Technology). We would like to appreciate Dr. Motoaki Hara and Dr. Yuichiro Yano.

\section{REFERENCE}

1. Knappe, S. et al. A microfabricated atomic clock. Applied Physics Letters 85 , 1460-1462 (2004).

2. Knappe, S. et al. A microfabricated atomic clock. Applied Physics Letters 85 , 1460-1462 (2004).

3. Knappe, S. MEMS Atomic Clocks. in Comprehensive Microsystems vol. 3 571612 (Elsevier, 2008).

4. Hasegawa, M. et al. Microfabrication of cesium vapor cells with buffer gas for MEMS atomic clocks. Sensors and Actuators, A: Physical 167, 594-601 (2011).

5. Chutani, R. et al. Laser light routing in an elongated micromachined vapor cell with diffraction gratings for atomic clock applications. Scientific Reports 5, 1-12 (2015).

6. Ender, H. et al. Fiber-Optic Time Transfer for UTC-Traceable Synchronization for Telecom Networks. IEEE Communications Standards Magazine 1, 66-73 (2017).

7. Lin, J. C. Synchronization Requirements for 5G: An Overview of Standards and Specifications for Cellular Networks. IEEE Vehicular Technology Magazine 13, 91-99 (2018). 
8. Hara, M. et al. Microwave oscillator using piezoelectric thin-film resonator aiming for ultraminiaturization of atomic clock. Review of Scientific Instruments $\mathbf{8 9}$, 105002 (2018).

9. H. Zhang, H. Herdian, A. T. Narayanan, A. Shirane, M. Suzuki, K. Harasaka, K. Adach, S. Yanagimachi, K. O. Ultra-Low-Power Atomic Clock for Satellite Constellation with $2.2 \times 10$-12 Long-Term Allan Deviation Using Cesium Coherent Population Trapping. ISSCC Dig. Tech. Papers 462-464 (2019).

10. Nishino, H. et al. Reflection-type vapor cell for micro atomic clocks using local anodic bonding of $45^{\circ}$ mirrors. Optics Letters 46, (2021).

11. Vanier, J. \& Audoin, C. The Quantum Physics of Atomic Frequency Standards. (Adam Hilger, Bristol and Philadelphia, 1989).

12. Townes, C. H. Atomic clocks and frequency stabilization on microwave spectral lines. Journal of Applied Physics 22, 1365-1372 (1951).

13. Katori, H. Optical lattice clocks and quantum metrology. Nature Photonics $\mathbf{5}$, 203-210 (2011).

14. Hachisu, H., Nakagawa, F., Hanado, Y. \& Ido, T. Months-long real-time generation of a time scale based on an optical clock. Scientific Reports 8, 1-12 (2018).

15. Shah, V., Knappe, S., Schwindt, P. D. D. \& Kitching, J. Subpicotesla atomic magnetometry with a microfabricated vapour cell. 649-652 (2007)

doi:10.1038/nphoton.2007.201.

16. Kitching, J., Knappe, S., Shah, V., Griffith, C. \& Jimenez, R. Microfabricated Atomic Magnetometers and Applications. 789-794 (2008).

17. Kitching, J., Knappe, S. \& Donley, E. A. Atomic Sensors - A Review. 11, 1749$1758(2011)$.

18. Knappe, S., Alem, O., Sheng, D. \& Kitching, J. Microfabricated OpticallyPumped Magnetometers for Biomagnetic Applications. Journal of Physics: Conference Series 723, (2016). 
19. Noor, R. M. \& Shkel, A. M. MEMS components for NMR atomic sensors. Journal of Microelectromechanical Systems 27, 1148-1159 (2018).

20. Petersen, K. E. Silicon as a mechanical material. Proceedings of the IEEE 70, (1982).

21. Esashi, M. Wafer level packaging of MEMS. Journal of Micromechanics and Microengineering 18, 073001 (2008).

22. Esashi, M. \& Ono, T. From MEMS to nanomachine. Journal of Physics D: Applied Physics 38, (2005).

23. Wallis, G. \& Pomerantz, D. I. Field assisted glass-metal sealing. Journal of Applied Physics 40, 3946-3949 (1969).

24. Knapkiewicz, P. Technological assessment of MEMS alkali vapor cells for atomic references. Micromachines vol. 10 (2018).

25. Knappe, S. et al. Atomic vapor cells for chip-scale atomic clocks with improved long-term frequency stability.

26. Douahi, A. et al. New vapor cell technology for chip scale atomic clock.

Proceedings of the IEEE International Frequency Control Symposium and Exposition 58-61 (2007) doi:10.1109/FREQ.2007.4319031.

27. Douahi, A. et al. Vapour microcell for chip scale atomic frequency standard. Electronics Letters 43, 33-34 (2007).

28. Bopp, D. G., Maurice, V. M. \& Kitching, J. E. Wafer-level fabrication of alkali vapor cells using in-situ atomic deposition. JPhys Photonics 3, (2021).

29. Vanier, J., Godone, A. \& Levi, F. Coherent population trapping in cesium: Dark lines and coherent microwave emission. Physical Review A 58, 2345-2358 (1998).

30. Bernheim, R. A. Spin Relaxation in Optical Pumping. The Journal of Chemical Physics 36, 135-140 (1962).

31. Yano, Y., Kajita, M., Ido, T. \& Hara, M. Coherent population trapping atomic clock by phase modulation for wide locking range. Applied Physics Letters 111, (2017). 
32. Allan, D. W. Statistics of Atomic Frequency Standards. Proceedings of the IEEE 54, 221-230 (1966).

33. Corney, A. Atomic and Laser Spectroscopy. (Oxford, 1977). doi:10.1093/acprof:oso/9780199211456.001.0001.

34. Lin, C. W., Hsu, C. P., Yang, H. A., Wang, W. C. \& Fang, W. Implementation of silicon-on-glass MEMS devices with embedded through-wafer silicon vias using the glass reflow process for wafer-level packaging and 3D chip integration. Journal of Micromechanics and Microengineering 18, (2008).

35. Van Toan, N., Sangu, S. \& Ono, T. Glass reflow process for microsystem applications. Journal of Micromechanics and Microengineering 26, (2016).

36. Harz, M. \& Brückner, W. Stress Reduction in Anodically Bonded Silicon and Borosilicate Glass by Thermal Treatment. Journal of The Electrochemical Society 143, (1996).

37. Harz, M. \& Engelke, H. Curvature changing or flattening of anodically bonded silicon and borosilicate glass. Sensors and Actuators A: Physical 55, (1996).

38. Siddons, P., Adams, C. S., Ge, C. \& Hughes, I. G. Absolute absorption on rubidium D lines: Comparison between theory and experiment. Journal of Physics B: Atomic, Molecular and Optical Physics 41, (2008).

39. K. Shimoda. Introduction to Laser Physics, Springer Series in Optical Sciences. vol. 44 (Springer, Berlin, 1984). 


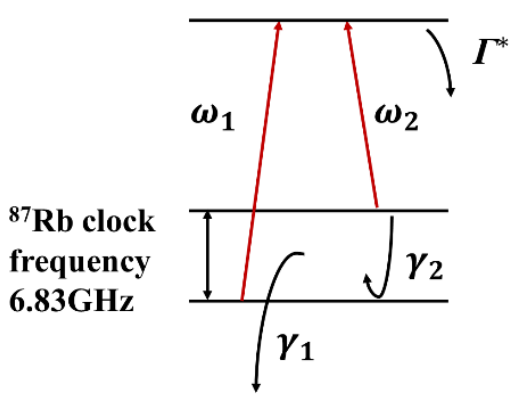

(a)

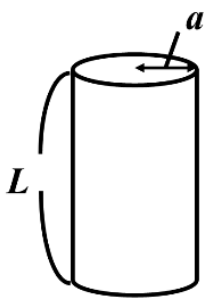

(b)

Figure 1

(a) Energy levels about CPT resonance. $\omega_{1}$ and $\omega_{2}$ are the laser angular frequencies from two sidebands of VCSEL. $\gamma_{1}$ and $\gamma_{2}$ are the ground-state relaxation rates of the populations and of the coherence, respectively. $\Gamma^{*}$ is the decay rate of the excited state. (b) Structure model of the vapor cell. $a$ is radius and $L$ is length of the cylindrical column. 


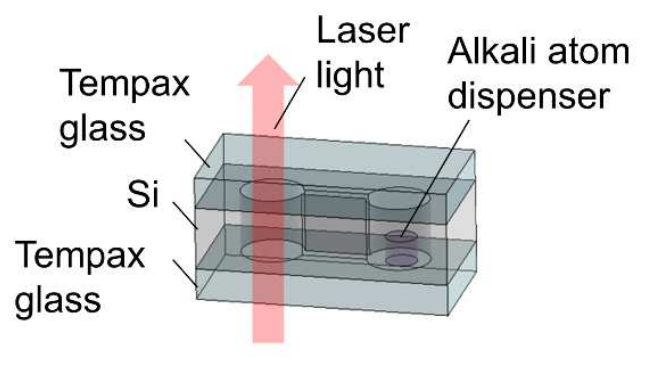

(a)

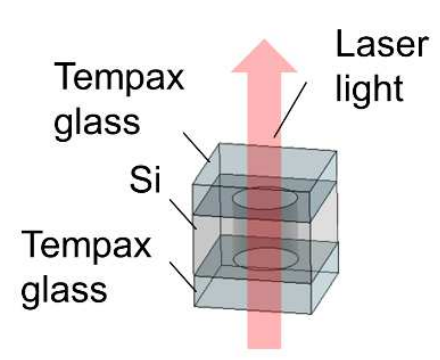

(b)

Figure 2

(a) A vapor cells using alkali atom dispenser. This vapor cell is fabricated by conventional fabrication method, which remains a dispenser.

(b) a vapor cell fabricated by proposal fabrication method, and this is also used an alkali atom dispenser, but the dispenser is separated away in the fabrication process. 
1. Si holes by a mechanical drill

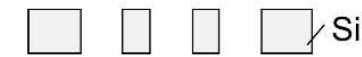

2. Anodic bonding

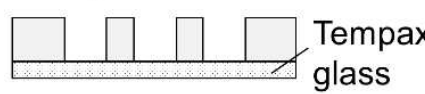

3. Anodic bonding Tempax glass

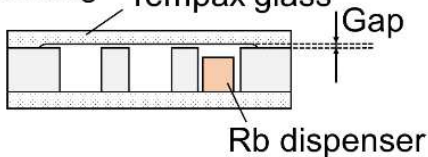

4. Activation of $\mathrm{Rb}$ dispenser, and anodic bonding

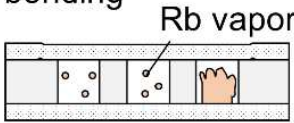

5. Dicing

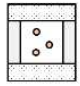

(a)

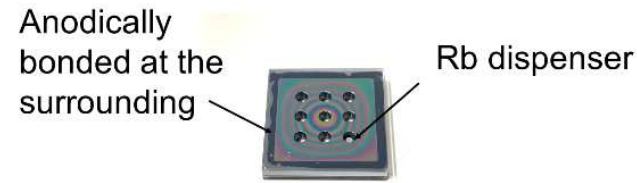

(b)

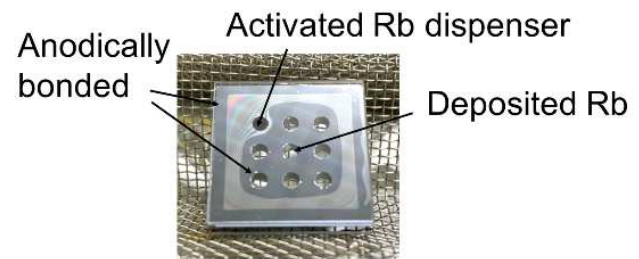

(c)

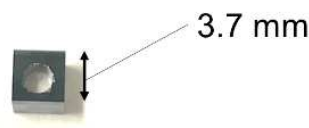

(d)

Figure 3

(a)Process chart of the proposal vapor cell using the $\mathrm{Rb}$ atom dispenser. Process 3 is sealing process in vacuum or in controlled pressure with the buffer gases.

(b) After Process 3, only the surrounding was anodically bonded in $20 \mathrm{~mm}$ square wafer.

(c) After Process 4, the center part was also anodically bonded, and some cells were observed deposited $\mathrm{Rb}$ at the glass surface of the holes.

(d) After Process 5, the vapor cell size is $3.7 \times 3.7 \times 4.0 \mathrm{~mm}$. 


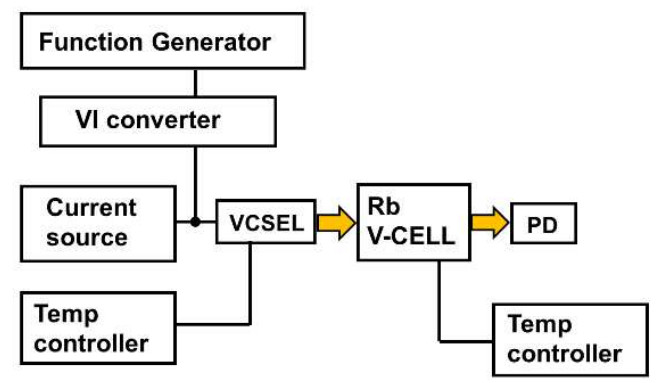

(a)

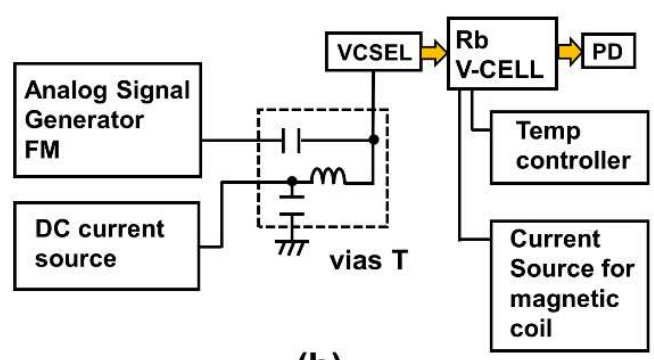

(b)

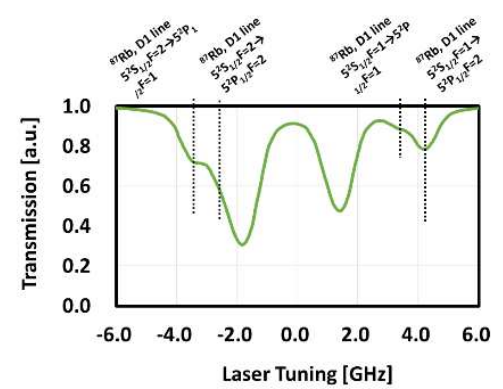

(c)

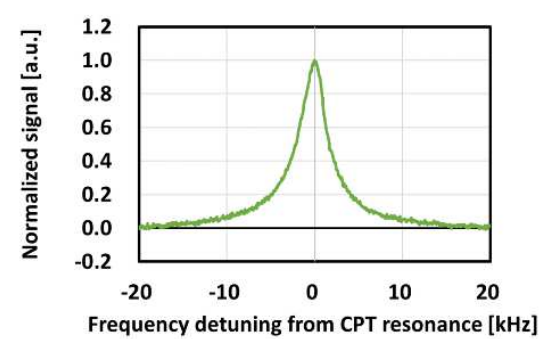

(d)

Figure 4

(a) Measurement setup for the absorption line spectrum. VCSEL is used wavelength 795 $\mathrm{nm}$, and applied current is added DC vias and modulation current.

(b) Measurement setup for the CPT resonance. Analog signal Generator output 3.417 $\mathrm{GHz}$, which is a half of the clock transition frequency.

(c) Absorption line spectrum lines of Rb D1 line of the fabricated vapor cell. The measurement temperature was $90{ }^{\circ} \mathrm{C}$ and the setup was described in (a). The cell was sealed in vacuum, but the absorption lines were broadened.

(d) CPT resonance of the fabricated vapor cell. The measurement temperature was $90{ }^{\circ} \mathrm{C}$ and the setup was described in (b). FWHM of the peak was $4.3 \mathrm{kHz}$ and the peak was shifted $4.7 \mathrm{kHz}$ from the transition frequency for $87 \mathrm{Rb}$. 


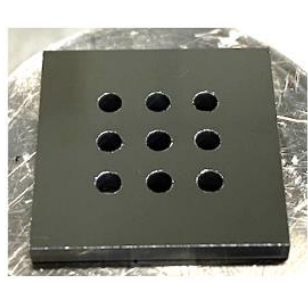

(a)

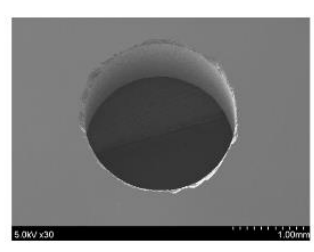

(b)

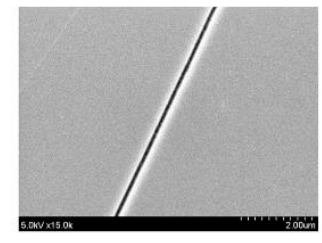

(c)

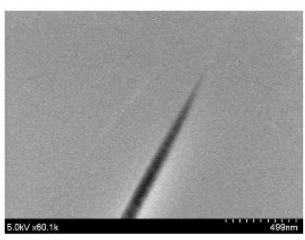

(d)
Scratches

penetrating the air

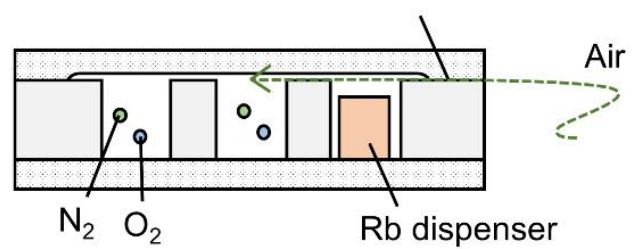

(e)

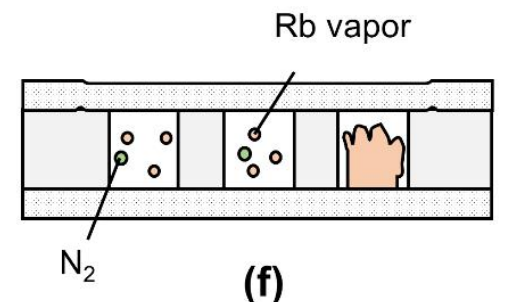

Figure 5

(a) The $20 \mathrm{~mm}$ square wafer after Process 1 in Fig. 3.

(b) SEM image of the hole, which is processed by mechanical drills.

(c) and (d) SEM image of the scratches on the Si surface.

(e) The expected image of leaking through the scratches on the bonded layer

(f) The expected image after Process 4 in Fig. 3, and $\mathrm{N}_{2}$ could remain but $\mathrm{O}_{2}$ could be absorbed by $\mathrm{Rb}$ dispenser. 


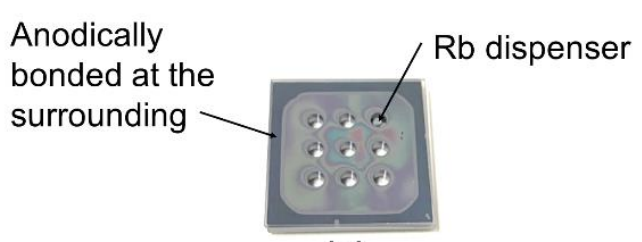

(a)

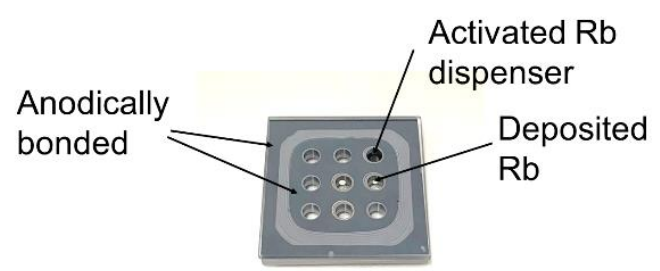

(b)

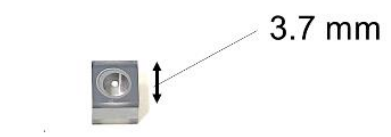

(c)

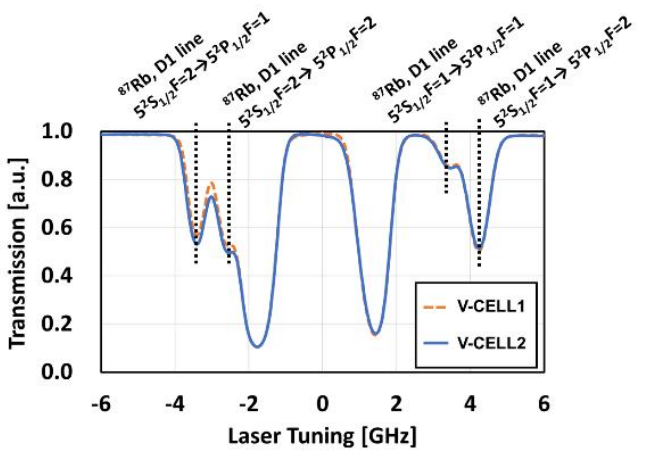

(d)

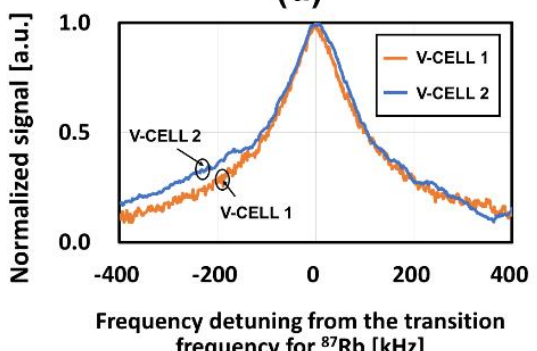

(e)

Figure 6

(a) After Process 3 in Fig. 3, only the surrounding was anodically bonded and vacuum sealed in $20 \mathrm{~mm}$ square wafer.

(b) After Process 4 in Fig. 3, the center part was also anodically bonded, and some cells were observed deposited $\mathrm{Rb}$ on the surface of the glass.

(c) After Process 5 in Fig. 3, the vapor cell size is $3.7 \times 3.7 \times 4.0 \mathrm{~mm}$, which cell name is V-CELL 1. On the center part of the glass surface deposited Rb was found.

(d) Absorption line spectrum of Rb D1 line of the fabricated vapor cells, which were named V-CELL 1 and V-CELL 2. They were fabricated in the same wafer and sealed in vacuum. The measurement temperature was $90{ }^{\circ} \mathrm{C}$ and the setup was described in Fig. 4 (a).

(e) The measurement results of the CPT resonance of the fabricated vapor cells. The measurement temperature was $90{ }^{\circ} \mathrm{C}$ and the setup was described in Fig. 4 (b). FWHM of the peak were $190 \mathrm{kHz}$ and $220 \mathrm{kHz}$, respectively. 


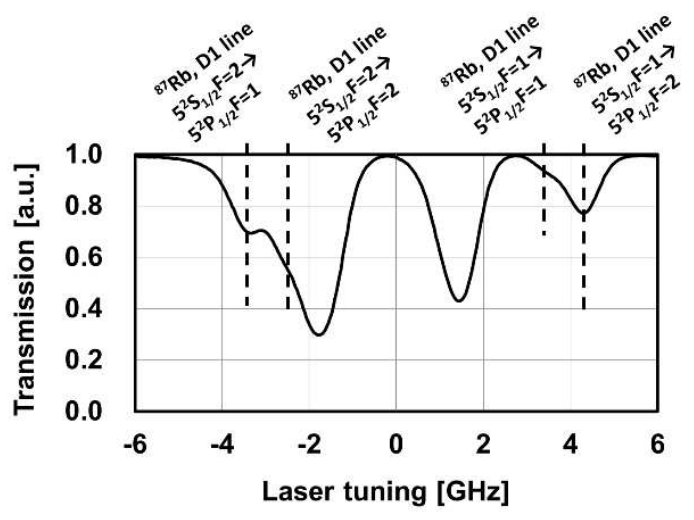

(a)

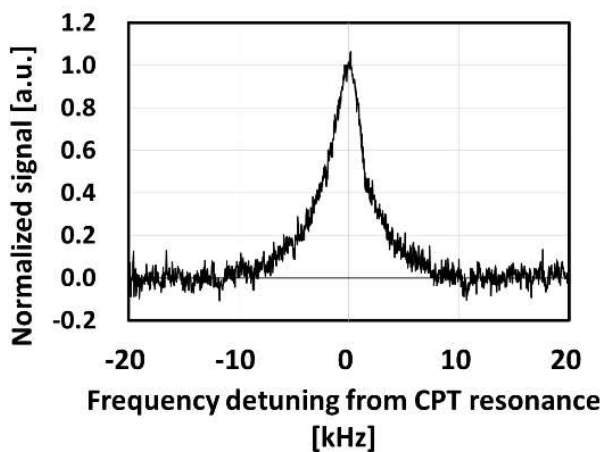

(b)

Figure 7

(a) Absorption line spectrum of Rb D1 line of the fabricated vapor cell, and the cell was sealed with $\mathrm{N}_{2} / \mathrm{Ar}$ buffer gases. The measurement temperature was $90{ }^{\circ} \mathrm{C}$ and the setup was described in Fig. 4 (a).

(b) CPT resonance of the fabricated vapor cell, and the cell was sealed with $\mathrm{N}_{2} /$ Ar buffer gases. The measurement temperature was $90{ }^{\circ} \mathrm{C}$ and the setup was described in Fig. 4 (b). FWHM of the peak was $4.0 \mathrm{kHz}$. 